\title{
Multi-integrals of finite variation
}

\section{Domenico Candeloro $^{1}$ (D) - Luisa Di Piazza ${ }^{2}$ (D) Kazimierz Musiał $^{3}$ (D) Anna Rita Sambucini ${ }^{1}$}

This work concludes a research cycle, but not the friendship that has tied us. You left us, dear Mimmo, too soon. The disease has won, but your memories will always be with us.

Received: 2 December 2019 / Accepted: 14 January 2020

(c) Unione Matematica Italiana 2020

This research was partially supported by Grant "Metodi di analisi reale per l'approssimazione attraverso operatori discreti e applicazioni” (2019) of GNAMPA - INDAM (Italy), by University of Perugia - Fondo Ricerca di Base 2019 "Integrazione, Approssimazione, Analisi Nonlineare e loro Applicazioni”, by University of Palermo - Fondo Ricerca di Base 2019 and by Progetto Fondazione Cassa di Risparmio cod. nr. 2018.0419.021 (title: Metodi e Processi di Intelligenza artificiale per lo sviluppo di una banca di immagini mediche per fini diagnostici (B.I.M.)).

$\bowtie$ Luisa Di Piazza

luisa.dipiazza@unipa.it

Extended author information available on the last page of the article 
Finally, in the last section, using $D L$ or $D b$ conditions we are able to prove that the scalar integrability of a multifunction can be obtained as a translation of the Pettis integrability (Theorem 4.1), while its Henstock integrability under $D L$ condition is obtained using Birkhoff integrability (Theorem 4.3), both results with integrals of finite variation.

This article is the last in which Domenico Candeloro was able to cooperate and to give his personal contribution, always precious, and we want to dedicate it to him, in his memory.

\section{Preliminaria}

Throughout $X$ is a Banach space with norm $\|\cdot\|$ and its dual $X^{*}$. The closed unit ball of $X$ is denoted by $B_{X}$. The symbol $c(X)$ denotes the collection of all nonempty closed convex subsets of $X$ and $c b(X), c w k(X)$ and $c k(X)$ denote respectively the family of all bounded, weakly compact and compact members of $c(X)$. For every $C \in c(X)$ the support function of $C$ is denoted by $s(\cdot, C)$ and defined on $X^{*}$ by $s\left(x^{*}, C\right)=\sup \left\{\left\langle x^{*}, x\right\rangle: x \in C\right\}$, for each $x^{*} \in X^{*} .\|C\|_{h}=d_{H}(C,\{0\}):=\sup \{\|x\|: x \in C\}$ and $d_{H}$ is the Hausdorff metric on the hyperspace $c b(X)$. The map $i: c b(X) \rightarrow \ell_{\infty}\left(B_{X^{*}}\right)$ given by $i(A):=s(\cdot, A)$ is the Rådström embedding (see, for example, [1, Theorem 3.2.9 and Theorem 3.2.4(1)], [14, Theorem II-19], or [28]).

$\mathcal{I}$ is the collection of all closed subintervals of the unit interval $[0,1]$. All functions investigated are defined on the unit interval $[0,1]$ endowed with Lebesgue measure $\lambda$ and Lebesgue measurable sets $\mathcal{L}$.

A map $\Gamma:[0,1] \rightarrow c(X)$ is called a multifunction. In the sequel, given a multifunction $\Gamma:[0,1] \rightarrow c(X)$, we set $D_{\Gamma}(t):=\operatorname{diam}(\Gamma(t))$, for all $t \in[0,1]$. We say that $\Gamma$ satisfies the

\section{$(D b$-condition $) \quad$ if $\sup ^{\operatorname{ess}} D_{\Gamma}(t)<\infty$;}

(DL-condition) if $\int_{0}^{1} D_{\Gamma}(t) d t<+\infty$ (where $\int$ denotes the upper integral).

We recall that a multifunction $\Gamma:[0,1] \rightarrow c(X)$ is said to be integrably bounded if there is a function $h \in L_{1}[0,1]$ such that $\|\Gamma(t)\|_{h} \leq|h(t)|$ for almost all $t \in[0,1]$. We have always $D_{\Gamma}(t) \leq 2\|\Gamma(t)\|_{h}$. Hence, if $\Gamma$ is integrably bounded, then $\Gamma$ satisfies $D L$.

If $\Gamma(t) \ni 0$ for almost every $t \in[0,1]$, then $\|\Gamma(t)\|_{h} \leq D_{\Gamma}(t)$ a.e. Each function $g:[0,1] \rightarrow X$, considered as a $c k(X)$-valued multifunction, trivially satisfies the $D b$ property.

We recall that if $\Phi: \mathcal{L} \rightarrow Y$ is an additive vector measure with values in a normed space $Y$, then the variation of $\Phi$ is the extended non negative function $|\Phi|$ whose value on a set $E \in \mathcal{L}$ is given by $|\Phi|(E)=\sup _{\pi} \sum_{A \in \pi}\|\Phi(A)\|$, where the supremum is taken over all partitions $\pi$ of $E$ into a finite number of pairwise disjoint members of $\mathcal{L}$. If $|\Phi|<\infty$, then $\Phi$ is called a measure of finite variation.

If the measure $\Phi$ is defined only on $\mathcal{I}$, the finite partitions considered in the definition of variation are composed by intervals. In this case we will speak of finite interval variation and we will use the symbol $\widetilde{\Phi}$, namely:

$$
\widetilde{\Phi}([0,1])=\sup \left\{\sum_{i}\left\|\Phi\left(I_{i}\right)\right\|:\left\{I_{1}, \ldots, I_{n}\right\} \text { is a finite interval partition of }[0,1]\right\} .
$$

If $Y$ is a metric space, for example $\left(c b(X), d_{H}\right)$, which is a near vector space in the sense of [28], and $\Phi: \mathcal{I} \rightarrow c b(X)$ is additive we consider in its interval variation the distance $d_{H}(\Phi(A),\{0\})$ instead of $\|\Phi(A)\|$. 
We recall here briefly the definitions of integrals involved in this article. A scalarly integrable multifunction $\Gamma:[0,1] \rightarrow c(X)$ is Dunford integrable in a non-empty family $\mathcal{C} \subset c\left(X^{* *}\right)$, if for every set $A \in \mathcal{L}$ there exists a set $M_{\Gamma}^{D}(A) \in \mathcal{C}$ such that

$$
s\left(x^{*}, M_{\Gamma}^{D}(A)\right)=\int_{A} s\left(x^{*}, \Gamma\right) d \lambda, \quad \text { for every } x^{*} \in X^{*} .
$$

If $M_{\Gamma}^{D}(A) \subset X$ for every $A \in \mathcal{L}$, then $\Gamma$ is called Pettis integrable. We write it as $(P) \int_{A} \Gamma d \mu$ or $M_{\Gamma}(A)$. We say that a Pettis integrable $\Gamma:[0,1] \rightarrow c(X)$ is strongly Pettis integrable, if $M_{\Gamma}$ is an $h$-multimeasure (i.e. it is countably additive in the Hausdorff metric).

A multifunction $\Gamma:[0,1] \rightarrow c b(X)$ is said to be Henstock (resp. McShane) integrable on $[0,1]$, if there exists $\Phi_{\Gamma}([0,1]) \in c b(X)$ with the property that for every $\varepsilon>0$ there exists a gauge $\delta:[0,1] \rightarrow \mathbb{R}^{+}$such that for each Perron partition (resp. partition) $\left\{\left(I_{1}, t_{1}\right), \ldots,\left(I_{p}, t_{p}\right)\right\}$ of $[0,1]$ with $I_{i} \subset\left[t_{i}-\delta\left(t_{i}\right), t_{i}+\delta\left(t_{i}\right)\right]$ for all $i$ (i.e. $\delta$-fine), we have

$$
d_{H}\left(\Phi_{\Gamma}([0,1]), \sum_{i=1}^{p} \Gamma\left(t_{i}\right) \lambda\left(I_{i}\right)\right)<\varepsilon .
$$

If the gauges above are taken to be measurable, then we speak of $\mathcal{H}$ (resp. Birkhoff)integrability on $[0,1]$. If $I \in \mathcal{I}$, then $\Phi_{\Gamma}(I):=\Phi_{\Gamma \chi_{I}}[0,1]$. Finally if, instead of formula (1), we have

$$
\sum_{i=1}^{p} d_{H}\left(\Phi_{\Gamma}\left(I_{i}\right), \Gamma\left(t_{i}\right) \lambda\left(I_{i}\right)\right)<\varepsilon
$$

we speak about variational Henstock (resp. McShane) integrability on $[0,1]$. In all the cases $\Phi_{\Gamma}: \mathcal{I} \rightarrow c b(X)$ is an additive interval multimeasure.

Thanks to the Rådström embedding, a multifunction $\Gamma$ is "gauge" integrable (in one of the previous types) if and only if its image $i \circ \Gamma$ in $l_{\infty}\left(B_{X^{*}}\right)$ is integrable in the same way.

A multifunction $\Gamma:[0,1] \rightarrow c b(X)$ is said to be Henstock-Kurzweil-Pettis (or HKP) integrable in $c b(X)$ if it is scalarly Henstock-Kurzweil (or HK)-integrable and for each $I \in \mathcal{I}$ there exists a set $N_{\Gamma}(I) \in c b(X)$ such that $s\left(x^{*}, N_{\Gamma}(I)\right)=(H K) \int_{I} s\left(x^{*}, \Gamma\right)$ for every $x^{*} \in X^{*}$. If an HKP-integrable $\Gamma$ is scalarly integrable, then it is called weakly McShane integrable (or wMS).

We recall that a function $f:[0,1] \rightarrow \mathbb{R}$ is Denjoy-Khintchine (DK) integrable ([25, Definition 11]), if there exists an ACG function (cf. [26]) F such that its approximate derivative is almost everywhere equal to $f$. A multifunction $\Gamma:[0,1] \rightarrow c b(X)$ is Denjoy-KhintchinePettis (DKP) integrable in a non empty family $\mathcal{C}$ in $c b(X)$, if for each $x^{*} \in X^{*}$ the function $s\left(x^{*}, \cdot\right)$ is Denjoy-Khintchine integrable and for every $I \in \mathcal{I}$ there exists $C_{I} \in \mathcal{C}$ with $(D K) \int_{I} s\left(x^{*}, \Gamma\right)=s\left(x^{*}, C_{I}\right)$, for every $x^{*} \in X^{*}$.

As regards other definitions of measurability and integrability that will be treated here and are not explained and the known relations among them, we refer to [3-7,9,10,13,17,21,31], in order do not burden the presentation. 


\section{Multimeasures of finite variation}

We begin with a known fact.

Lemma 3.1 If $f:[0,1] \rightarrow \mathbb{R}$ is the Denjoy-Khintchine integrable and the interval variation of its integral is finite, then $f$ Lebesgue integrable.

Proof Let $F$ be the Denjoy-Khintchine primitive of $f:[a, b] \rightarrow \mathbb{R}$. Then $F$ is an ACG function and, according to [26, Theorem 15.8], $F$ is continuous on $[a, b]$. So $F$ satisfies the condition $(\mathrm{N})$ of Lusin on in $[a, b]$ (see [26, Theorem 6.12]). Since $F$ is also BV, an application of [26, Theorem 6.15] gives that $F$ is also AC on on $[a, b]$. So $f$ is Lebesgue integrable.

Theorem 3.2 Let $\Phi: \mathcal{I} \rightarrow c b(X)$ be the DKP-integral of $\Gamma:[0,1] \rightarrow c b(X)$. If $\sup _{x^{*} \in B_{X}}\left\langle\widehat{\left\langle x^{*}, \Phi\right\rangle}([0,1])<\infty\right.$, then $\Gamma$ is weakly McShane integrable in $c b(X)$ and Gelfand integrable in $c w^{*} k\left(X^{* *}\right)$. If $\widetilde{\Phi}([0,1])<\infty$, then $\Phi$ is strongly Pettis integrable in cb $(X)$.

Proof By Lemma 3.1 $\Gamma$ is wMS-integrable in $c b(X)$. According to [8, Theorem 3.2] it is Gelfand integrable in $c w^{*} k\left(X^{* *}\right)$. Denote the Gelfand integral by $\Psi$.

Assume now that $\widetilde{\Phi}([0,1])<\infty$. If $\left\{I_{i}: i \in \mathbb{N}\right\}$ is a sequence of non-overlapping subintervals of $[0,1]$, then

$$
\sum_{i}\left\|\Phi\left(I_{i}\right)\right\|_{h} \leq \widetilde{\Phi}([0,1])<\infty
$$

and so, due to the completeness of $c b(X)$ under Hausdorff distance, the series $\sum_{i} \Phi\left(I_{i}\right)$ is convergent in $c b(X)$.

But for each $x^{*} \in X^{*}$ the function $s\left(x^{*}, \Psi\right)$ is a measure and so $\sum_{i} s\left(x^{*}, \Phi\left(I_{i}\right)\right)=$ $s\left(x^{*}, \Psi\left(\bigcup_{i} I_{i}\right)\right)$. Since the sum of $\sum_{i} \Phi\left(I_{i}\right)$ is uniquely determined, we have

$$
\Psi\left(\bigcup_{i} I_{i}\right)=\sum_{i} \Phi\left(I_{i}\right) \in c b(X) .
$$

It follows that $\Psi$ is $\sigma$-additive (in the Hausdorff metric) on the algebra $\mathfrak{J}$ generated by $\mathcal{I}$. Hence, also $i \circ \Psi$ is $\sigma$-additive on $\mathfrak{J}$. But $\widetilde{i \circ \Psi}([0,1])=\widetilde{\Psi}([0,1])=\widetilde{\Phi}([0,1])<\infty$ and so due to [15, Proposition I.15], $i \circ \Psi$ restricted to $\mathfrak{J}$ is strongly additive.

It is a consequence of [27] or [15, Theorem I.5.2] that $i \circ \Psi$ is a measure on the $\sigma$-algebra of Borel subsets of [0, 1]. But $i \circ \Psi(E)=0$, provided Lebesgue measure vanishes on $E$ and consequently, $i \circ \Psi$ is measure on $\mathcal{L}$. Since $i(c b(X))$ is a closed cone also $\Psi$ is a measure in the Hausdorff metric of $c b(X)$ and therefore $\Gamma$ is strongly Pettis integrable on $\mathcal{L}$.

Corollary 3.3 If $\Gamma:[0,1] \rightarrow c(X)$ is Pettis integrable in $c b(X), M_{\Gamma}$ is its indefinite Pettis integral and $\left|M_{\Gamma}\right|([0,1])<\infty$, then $\Gamma$ is strongly Pettis integrable.

Proof It is easily seen that due to the finite variation of $M_{\Gamma}$, the multifunction $\Gamma$ takes a.e. bounded values. Without loss of generality we may assume that $\Gamma:[0,1] \rightarrow c b(X)$. We have $\widetilde{M_{\Gamma}}([0,1]) \leq\left|M_{\Gamma}\right|([0,1])<\infty$ and so we may apply Theorem 3.2.

Under stronger assumptions one obtains stronger results. We proved in [8] the following

Theorem 3.4 Let $\Gamma:[0,1] \rightarrow c b(X)$ be Henstock $($ or $\mathcal{H})$ integrable and let $\Phi_{\Gamma}$ be its Henstock $\left(\mathcal{H}\right.$ )-integral. If $\widetilde{\Phi}_{\Gamma}[0,1]<\infty$, then $\Gamma$ is McShane (Birkhoff) integrable. 
Finally, we can formulate the characterization of variationally McShane integral in terms of the variational Henstock integral.

Theorem 3.5 A multifunction $\Gamma:[0,1] \rightarrow c b(X)$ is variationally McShane integrable if and only if it is variationally Henstock integrable and the interval variation of the Henstock integral is finite.

Proof We need to prove only that each $\mathrm{vH}$-integrable multifunction $\Gamma:[0,1] \rightarrow c b(X)$ with integral of finite interval variation is variationally McShane integrable. We know already from Theorem 3.2 that $\Gamma$ is Pettis integrable. Since $i \circ \Gamma$ is vH-integrable it is strongly measurable. If $M_{\Gamma}$ is the Pettis integral of $\Gamma$, then $i \circ M_{\Gamma}$ is a measure of finite variation and $i \circ M_{\Gamma}(I)=(v H) \int_{I} i \circ \Gamma$. It follows that $i \circ \Gamma$ is Bochner integrable. Now we may apply [5, Proposition 3.6] to obtain variational McShane integrability of $\Gamma$.

In case of vector valued functions $f:[0,1] \rightarrow X$, by the properties of the Pettis and the Bochner integrals, it follows at once that if $f$ is strongly measurable, Pettis integrable and its Pettis integral has finite variation, then $f$ is Bochner integrable. The next result is the multivalued version of this result.

Theorem 3.6 Let $\Gamma:[0,1] \rightarrow c b(X)$ be Bochner measurable, Pettis integrable, and its Pettis integral has finite variation. Then $\Gamma$ is integrably bounded.

Proof Since $\Gamma$ is Bochner measurable, it is a.e. limit of simple multifunctions. It follows that $i \circ \Gamma$ is strongly measurable. Let us assume that $Y:=\overline{\operatorname{span}}(i \circ \Gamma([0,1]))$ is a closed separable subspace of $\ell_{\infty}\left(B_{X^{*}}\right)$. Then, we follow the proof of [12, Proposition 3.5]. If $e_{x^{*}} \in B_{\ell_{\infty}\left(B_{X^{*}}\right)^{*}}$ is defined by $\left\langle e_{x^{*}}, g\right\rangle:=g\left(x^{*}\right)$ for every $g \in \ell_{\infty}\left(B_{X^{*}}\right)$, then the set $B:=\left\{e_{x^{*}} \mid Y: x^{*} \in\right.$ $\left.B_{X^{*}}\right\} \subset B_{Y^{*}}$ is norming. By the Pettis integrability of $\Gamma$ the family $\mathcal{Z}_{i \circ \Gamma, B}:=\left\{\left\langle e_{x^{*}}, i \circ \Gamma\right\rangle\right.$ : $\left.x^{*} \in B_{X^{*}}\right\}=\left\{s\left(x^{*}, \Gamma\right): x^{*} \in B_{X^{*}}\right\}$ is uniformly integrable. Consequently, $i \circ \Gamma$ is a Pettis integrable function. Moreover, $i\left((P) \int_{A} \Gamma d \lambda\right)=(P) \int_{A} i \circ \Gamma d \lambda$ for every $A \in \mathcal{L}$ (see the proof of [12, Proposition 3.5]). By the assumption the variation of $(P) \int i \circ \Gamma d \lambda$ is finite and so $i \circ \Gamma$ is Bochner integrable. Consequently, $\Gamma$ is integrably bounded.

Then by [5, Proposition 3.6] (formulated for $c b(X)$-valued multifunctions) and Theorem 3.6 we get the following

Proposition 3.7 Let $\Gamma:[0,1] \rightarrow c b(X)$ be a scalarly measurable multifunction. Then the following conditions are equivalent:

1. $\Gamma$ is variationally McShane integrable;

2. $i(\Gamma) \in L_{1}\left(\lambda, \ell_{\infty}\left(B_{X^{*}}\right)\right)$;

3. $\Gamma$ is Bochner measurable and integrably bounded;

4. $\Gamma$ is Bochner measurable, Pettis integrable, and its Pettis integral has finite variation.

Proof It is an immediate consequence of Theorem 3.6 if we proceed analogously to [5, Proposition 3.6].

\section{Decompositions}

In the study of the integrability of multifunctions it is important to decompose a multifunction as a sum of a selection that is integrable in the same sense and a multifunction that is integrable in a stronger sense than the original one (see for example $[5-8,18,19,24]$ ). Using $D b$ or $D L$ conditions we are able to extend decomposition results and to write integrable multifunctions as a translation of a multifunction with its integral of finite variation. 
Theorem 4.1 Let $\Gamma:[0,1] \rightarrow c(X)$ be integrable in $c b(X)(c w k(X)$ or $c k(X))$ in the sense of one of the scalarly defined integrals. If $\Gamma$ possesses at least one selection integrable in the same way, then the following conditions are equivalent:

1. $\Gamma$ satisfies the DL-condition (or Db condition);

2. $\Gamma=G+f$, where $f$ is a properly integrable selection of $\Gamma, G$ is Pettis integrable in $\operatorname{cb}(X)(\operatorname{cwk}(X)$ or $c k(X))$ and $\int_{0}^{1} D_{G}(t) d t<\infty$ (and $G$ is bounded). In particular the indefinite integral of $G$ is of finite variation.

Proof Assume that $\Gamma$ is DP-integrable. Due to [8, Theorem 3.5] $\Gamma=G+f$, where $G$ is Pettis integrable, $f$ is Denjoy integrable and $G$ satisfies the condition DL. It is obvious that the Pettis integral of $G$ is of finite variation.

We observe that in Theorem 4.1 the multifunctions are arbitrary, in particular they may take weakly locally compact values that do not contain any line, but the thesis is still the same.

Remark 4.2 Unfortunately, even if $G:[0,1] \rightarrow c k(X)$ is a positive multifunction that is Pettis integrable and its integral is of finite variation, the multifunction $G$ may not satisfy the DL condition. To see it let $X=\ell_{2}[0,1]$ and let $\left\{e_{t}: t \in(0,1]\right\}$ be its orthonormal system. If $G(t):=\operatorname{conv}\left\{0, e_{t} / t\right\}$, then $s(x, G)=0$ a.e. for each separate $x \in \ell_{2}[0,1]$ and so the integral and its variation are equal zero. However, $\operatorname{diam}\{G(t)\}=1 / t$ and so the DL-condition fails. Moreover, $G$ is not Henstock integrable. Indeed, let $\delta$ be any gauge and $\left\{\left(I_{1}, t_{1}\right), \ldots,\left(I_{n}, t_{n}\right)\right\}$ be a $\delta$-fine Perron partition of $[0,1]$. Assume that $0 \in I_{1}$, then $t_{1} \leq\left|I_{1}\right|$. Hence $\lambda\left(I_{1}\right) / t_{1} \geq 1$ for $t_{1}>0$ and so $\left\|\sum_{i \leq n} \frac{e_{i}}{t_{i}} \lambda\left(I_{i}\right)\right\| \geq 1$. Consider now the multifunction given by $H(t):=\operatorname{con} v\left\{0, e_{t}\right\}$, where $X$ is as above. We are going to prove that $H$ is Birkhoff-integrable. Given $\varepsilon>0$, let $n \in \mathbb{N}$ be such that $1 / \sqrt{n}<\varepsilon$ and $\delta$ be any gauge, pointwise less than $1 / n$. If $\left\{\left(I_{1}, t_{1}\right), \ldots,\left(I_{m}, t_{m}\right)\right\}$ is a $\delta$-fine partition of $[0,1]$ and $\left\{J_{1}, \ldots, J_{n}\right\}$ is the division of $[0,1]$ into closed intervals of the same length, then

$$
\begin{aligned}
\left\|\sum_{i \leq m} e_{i} \lambda\left(I_{i}\right)\right\| & =\left\|\sum_{i \leq m} \sum_{k \leq n} e_{i} \lambda\left(I_{i} \cap J_{k}\right)\right\|=\left\|\sum_{k \leq n} \sum_{i \leq m} e_{i} \lambda\left(I_{i} \cap J_{k}\right)\right\| \\
& =\left(\sum_{k \leq n} \sum_{i \leq m} \lambda\left(I_{i} \cap J_{k}\right)^{2}\right)^{1 / 2} \leq 1 / \sqrt{n}<\varepsilon .
\end{aligned}
$$

[We apply here the inequality $\sum_{i} a_{i}^{2} \leq\left(\sum a_{i}\right)^{2}$. For each fixed $k \leq n$ we take as $a_{i}$ the number $\lambda\left(I_{i} \cap J_{k}\right)$ ]. If $\delta$ is measurable, then we get Birkhoff integrability of $H$.

Some additional results will be given now, in order to get decompositions with gauge integrable multifunctions.

Theorem 4.3 Let $\Gamma:[0,1] \rightarrow c w k(X)$ satisfy DL-condition, and assume that $\Gamma$ is $\mathcal{H}$ integrable (or H-integrable). Then we have $\Gamma=G+f$, where $f \in \mathcal{S}_{\mathcal{H}}(\Gamma)\left(f \in \mathcal{S}_{H}(\Gamma)\right)$ is arbitrary and $G$ is an abs-Birkhoff integrable multifunction. In particular the integral of $G$ has finite variation. If $\Gamma$ is Bochner measurable, then $G$ is also variationally Henstock integrable.

Proof Assume that $\Gamma$ is $\mathcal{H}$-integrable. It is known (see [20, Theorem 3.1]) that $\Gamma$ has an $\mathcal{H}$ integrable selection $f$. Thanks to [31, Theorem 4], both $i \circ \Gamma$ and $f$ are Riemann-measurable. So, if $G:=\Gamma-f$, it is clear that $i \circ G$ is Riemann-measurable too. Moreover, thanks to the 
$D L$-condition, the function $t \mapsto\|i \circ G(t)\|$ is integrably bounded, i.e. $\int_{0}^{1}\|i \circ G(t)\| d t<+\infty$ since $\|i \circ G(t)\|=\sup \left\{\|u\|_{X}: u \in G(t)\right\}=\sup \left\{\|v-f(t)\|_{X}: v \in \Gamma(t)\right\} \leq \operatorname{diam}(\Gamma(t))$. So, $i \circ G$ is Riemann-measurable and integrably bounded, which means that $i \circ G$ (and so $G$ ) is absolutely Birkhoff integrable, thanks to [11, Theorem 2].

Assume now that $\Gamma$ is $H$-integrable. Then, according to [8, Theorem 3.5] $\Gamma=G+f$, where $G$ is Birkhoff integrable. By the assumption $G$ satisfies the DL-condition. Hence again the function $t \mapsto\|i \circ G(t)\|$ is integrably bounded. Consequently, $i \circ G$ is absolutely Birkhoff integrable and hence also $G$. The vH-integrability of $G$ follows from [2, Corollary 4.1], since $G$ is Pettis integrable.

A similar result can be given also for Birkhoff integrable functions $\Gamma:[0,1] \rightarrow c w k(X)$ : the proof is essentially the same but instead of [20] we invoke [5, Theorem 3.4].

Proposition 4.4 Let $\Gamma:[0,1] \rightarrow c w k(X)$ satisfy DL-condition, and assume that $\Gamma$ is Birkhoff integrable. Then we have $\Gamma=G+f$, where $f$ is any Birkhoff integrable selection of $\Gamma$, and $G$ is an abs-Birkhoff integrable multifunction. In particular the integral of $G$ has finite variation.

Question 4.5 Assume that $f:[0,1] \rightarrow X$ is Birkhoff integrable and the classical variation of the indefinite integral is finite. Is $f$ absolutely Birkhoff integrable? That is, do we have $\int_{0}^{1}\|f(t)\| d t<\infty$ ? A partial answer is contained in [11, Corollary 2].

Another way, does there exist a Birkhoff integrable $f$ that is scalarly equivalent to zero and $\bar{\int}_{0}^{1}\|f(t)\| d t=\infty$ ? Recall that $G$ from Remark 4.2 is not Birkhoff integrable.

Fremlin proved that a Birkhoff integrable function is properly measurable in the Talagrand sense. It is known that $f$ is Talagrand integrable if and only if $f$ is properly measurable and $\bar{\int}\|f\| d \lambda<\infty$. Then, it is known that $f$ is absolutely Birkhoff integrable if and only if it is Riemann measurable and $\bar{\int}\|f\| d \lambda<\infty$ if and only if $f$ is Birkhoff integrable and $\bar{\int}\|f\| d \lambda<\infty$. Thus, if $f$ is absolutely Birkhoff integrable, then $f$ is also Talagrand integrable. The converse result fails by [22, Example 3C] (where a function $f:[0,1] \rightarrow$ $\ell_{\infty}(\mathbb{N})$ is shown, which is Talagrand but not even McShane integrable).

It is also possible to obtain decompositions where the multifunction $G$ turns out to be variationally McShane integrable, as follows.

Proposition 4.6 Let $\Gamma:[0,1] \rightarrow c w k(X)$ satisfy DL-condition, and assume that $\Gamma$ is Bochner measurable. Then we have $\Gamma=G+f$, where $f$ is any strongly measurable selection of $\Gamma$, and $G$ is a variationally McShane integrable multifunction.

Proof Let $f$ be any strongly measurable selection of $\Gamma$, and set $G=\Gamma-f$. Then clearly $G$ is Bochner measurable. Moreover, since $\Gamma$ satisfies the $D L$ condition and $f$ is a selection from $\Gamma, i \circ G$ is integrably bounded. Then $i \circ G$ is strongly measurable and integrably bounded, and therefore variationally McShane integrable. Of course this implies that also $G$ is integrable.

Proposition 4.7 If $\Gamma:[0,1] \rightarrow c w k(X)$ is Henstock $(\mathcal{H}$, variationally Henstock, Pettis, McShane, Birkhoff) integrable, then $\Gamma$ cannot be, in general, written as $G+f$, where $G$ is variationally McShane integrable and $f$ is integrable in the same way as $\Gamma$.

Proof Take $f$ as in [16]; then $f$ is $\mathrm{vH}$ and Pettis integrable, but not Bochner integrable. Let $\Gamma=\operatorname{conv}\{0, f(t)\}$, then $\Gamma$ is vH-integrable, Pettis but not Bochner integrable, as shown in 
[5, Example 4.7]. By [5, Theorem 4.3 (a) and (c)], then $\Gamma$ is also McShane integrable and then Birkhoff integrable, since $\Gamma$ is Bochner measurable. Following the same motivations of [6, Remark 5.4] the multifunction $G=\Gamma-f$ is not variationally McShane integrable.

Almost nothing is known on possible decompositions of a Pettis integrable multifunctions. We have only the following negative result:

Example 4.8 Let $\Gamma:[0,1] \rightarrow c w k(X)$ be Pettis integrable. Assume that $M_{\Gamma}(\mathcal{L})$ is not relatively compact in the Hausdorff metric. Then $\Gamma$ cannot be represented as $\Gamma=G+f$, where $G$ is McShane integrable and $f$ is a Pettis integrable selection of $\Gamma$. In such a case $i \circ \Gamma$ is not Pettis integrable.

Proof Suppose that such a decomposition exists. Then, since $G$ is McShane integrable, the function $i \circ G$ is also McShane integrable and consequently it has relatively norm compact range. That is however equivalent to the norm relative compactness of $M_{G}(\mathcal{L})$ in $d_{H}$. But then $G$ can be approximated by simple functions (see [30, Theorem 2.3]). Since the integral of $f$ is norm relatively compact (because Lebesgue measure is perfect) also $f$ can be approximated by simple functions in the Pettis norm (see [29, Theorem 9.1]). As a result the multifunction $\Gamma$ can be approximated by simple multifunctions, which is impossible, since its range $M_{\Gamma}(\mathcal{L})$ is not relatively compact in the Hausdorff metric. (see [30, Theorem 2.3]). The non-integrability of $i \circ \Gamma$ is a consequence of perfectness of Lebesgue measure. Indeed, the range of the integral of a Pettis integrable function on $[0,1]$ (or on any perfect measure space) is norm relatively compact $([24,3 \mathrm{~J}])$.

\section{References}

1. Beer, G.: Topologies on Closed and Closed Convex Sets, Mathematics and its Applications, vol. 268. Kluwer Academic Publishers Group, Dordrecht (1993)

2. Bongiorno, B., Di Piazza, L., Musiał, K.: A variational Henstock integral characterization of the RadonNikodym property. Ill. J. Math. 53(1), 87-99 (2009)

3. Candeloro, D., Croitoru, A., Gavrilut, A., Sambucini, A.R.: An extension of the Birkhoff integrability for multifunctions. Mediterr. J. Math. 13(5), 2551-2575 (2016). https://doi.org/10.1007/s00009-015-06397

4. Candeloro, D., Croitoru, A., Gavrilut, A., Iosif, A., Sambucini, A.R.: Properties of the Riemann-Lebesgue integrability in the non-additive case. Rend. Circ. Mat. Palermo II Ser. (2019). https://doi.org/10.1007/ s12215-019-00419-y

5. Candeloro, D., Di Piazza, L., Musiał, K., Sambucini, A.R.: Gauge integrals and selections of weakly compact valued multifunctions. J. Math. Anal. Appl. 441(1), 293-308 (2016). https://doi.org/10.1016/j. jmaa.2016.04.009

6. Candeloro, D., Di Piazza, L., Musiał, K., Sambucini, A.R.: Relations among gauge and Pettis integrals for multifunctions with weakly compact convex values. Annali di Matematica 197(1), 171-183 (2018). https://doi.org/10.1007/s10231-017-0674-z

7. Candeloro, D., Di Piazza, L., Musiał, K., Sambucini, A.R.: Some new results on integration for multifunction. Ricerche Mat. 67(2), 361-372 (2018). https://doi.org/10.1007/s11587-018-0376-X

8. Candeloro, D., Di Piazza, L., Musiał, K., Sambucini, A.R.: Integration of multifunctions with closed convex values in arbitrary Banach spaces. J. Convex Anal. 27(4) (2020) (to appear)

9. Candeloro, D., Di Piazza, L., Musiał, K., Sambucini, A.R.: Multifunctions determined by integrable functions. Int. J. Approx. Reason. 112, 140-148 (2019). https://doi.org/10.1016/j.ijar.2019.06.002

10. Candeloro, D., Sambucini, A.R.: Order-type Henstock and McShane integrals in Banach lattices setting, Sisy 20014- IEEE 12th International Symposium on Intelligent Systems and Informatics, Subotica-Serbia. https://doi.org/10.1109/SISY.2014.6923557 (2014)

11. Caponetti, D., Marraffa, V., Naralenkov, K.: On the integration of Riemann-measurable vector-valued functions. Monatshefte Math. 182, 513-536 (2017)

12. Cascales, C., Kadets, V., Rodriguez, J.: Birkhoff integral for multi-valued functions. J. Math. Anal. Appl. 297, 540-560 (2004) 
13. Cascales, B., Kadets, V., Rodriguez, J.: Measurable selectors and set-valued Pettis integral in nonseparable Banach spaces. J. Funct. Anal. 256, 673-699 (2009)

14. Castaing, C., Valadier, M.: Convex Analysis and Measurable Multifunctions. Lecture Notes Math, vol. 580. Springer, Berlin (1977)

15. Diestel, J., Uhl, J.J.: Vector Measures. Mathematical Surveys and Monographs, vol. 15. American Mathematical Society, Providence, RI (1977)

16. Di Piazza, L., Marraffa, V.: The McShane, PU and Henstock integrals of Banach valued functions. Czechoslov. Math. J. 52(3), 609-633 (2002)

17. Di Piazza, L., Marraffa, V., Satco, B.: Set valued integrability and measurability in non separable Fréchet spaces and applications. Math. Slovaca 66(5), 1119-1138 (2016)

18. Di Piazza, L., Musiał, K.: A characterization of variationally McShane integrable Banach-space valued function. Ill. J. Math. 45(1), 279-289 (2001)

19. Di Piazza, L., Musiał, K.: A decomposition of Denjoy-Khintchine-Pettis and Henstock-Kurzweil-Pettis integrable multifunctions, vector measures, integration and related topics. In: Curbera, G.P., Mockenhaupt, G., Ricker, W.J. (eds.) Operator Theory: Advances and Applications, vol. 201, pp. 171-182. Birkhauser Verlag, Basel (2010)

20. Di Piazza, L., Musiał, K.: Relations among Henstock, McShane and Pettis integrals for multifunctions with compact convex values. Monatshefte Math. 173, 459-470 (2014)

21. El Amri, K., Hess, C.: On the Pettis integral of closed valued multifunctions. Set Valued Anal. 8, 329-360 (2000)

22. Fremlin, D.H., Mendoza, J.: On the integration of vector-valued functions. Ill. J. Math. 38, 127-147 (1994)

23. Fremlin, D.H.: The Henstock and McShane integrals of vector-valued functions. Ill. J. Math. 38(3), 471-479 (1994)

24. Fremlin, D., Talagrand, M.: A decomposition theorem for additive set functions and applications to Pettis integral and ergodic means. Math. Z. 168, 117-142 (1979)

25. Gordon, R.A.: The Denjoy extension of the Bochner, Pettis and Dunford integrals. Stud. Math. XCII, 73-91 (1989)

26. Gordon, R.A.: The Integrals of Lebesgue, Denjoy, Perron and Henstock, Grad. Stud. Math., vol. 4. AMS, Providence (1994)

27. Katz, M.P.: On extension of vector measures. Sibirskij Mat. J. 13(5), 1158-1168 (1972). in Russian

28. Labuschagne, C.C.A., Pinchuck, A.L., van Alten, C.J.: A vector lattice version of Rådström's embedding theorem. Quaest. Math. 30(3), 285-308 (2007)

29. Musiał, K.: Topics in the theory of Pettis integration. Rend. Istit. Mat. Univ. Trieste 23, 177-262 (1991)

30. Musiał, K.: Approximation of Pettis integrable multifunctions with values in arbitrary Banach spaces. J. Convex Anal. 20(3), 833-870 (2013)

31. Naralenkov, K.M.: A Lusin type measurability property for vector-valued functions. J. Math. Anal. Appl. 417(1), 293-307 (2014)

Publisher's Note Springer Nature remains neutral with regard to jurisdictional claims in published maps and institutional affiliations.

\section{Affiliations}

\section{Domenico Candeloro ${ }^{1}$ (1) - Luisa Di Piazza ${ }^{2}$ (1) $\cdot$ Kazimierz Musiał ${ }^{3}$ (1) Anna Rita Sambucini ${ }^{1}$}

Domenico Candeloro

candelor@dmi.unipg.it

Kazimierz Musiał

kazimierz.musial@math.uni.wroc.pl

Anna Rita Sambucini

anna.sambucini@unipg.it

1 Department of Mathematics and Computer Sciences, University of Perugia 1, Via Vanvitelli, 06123

Perugia, Italy 
2 Department of Mathematics and Computer Sciences, University of Palermo, Via Archirafi 34, 90123 Palermo, Italy

3 Institute of Mathematics, Wrocław University, Pl. Grunwaldzki 2/4, 50-384 Wrocław, Poland

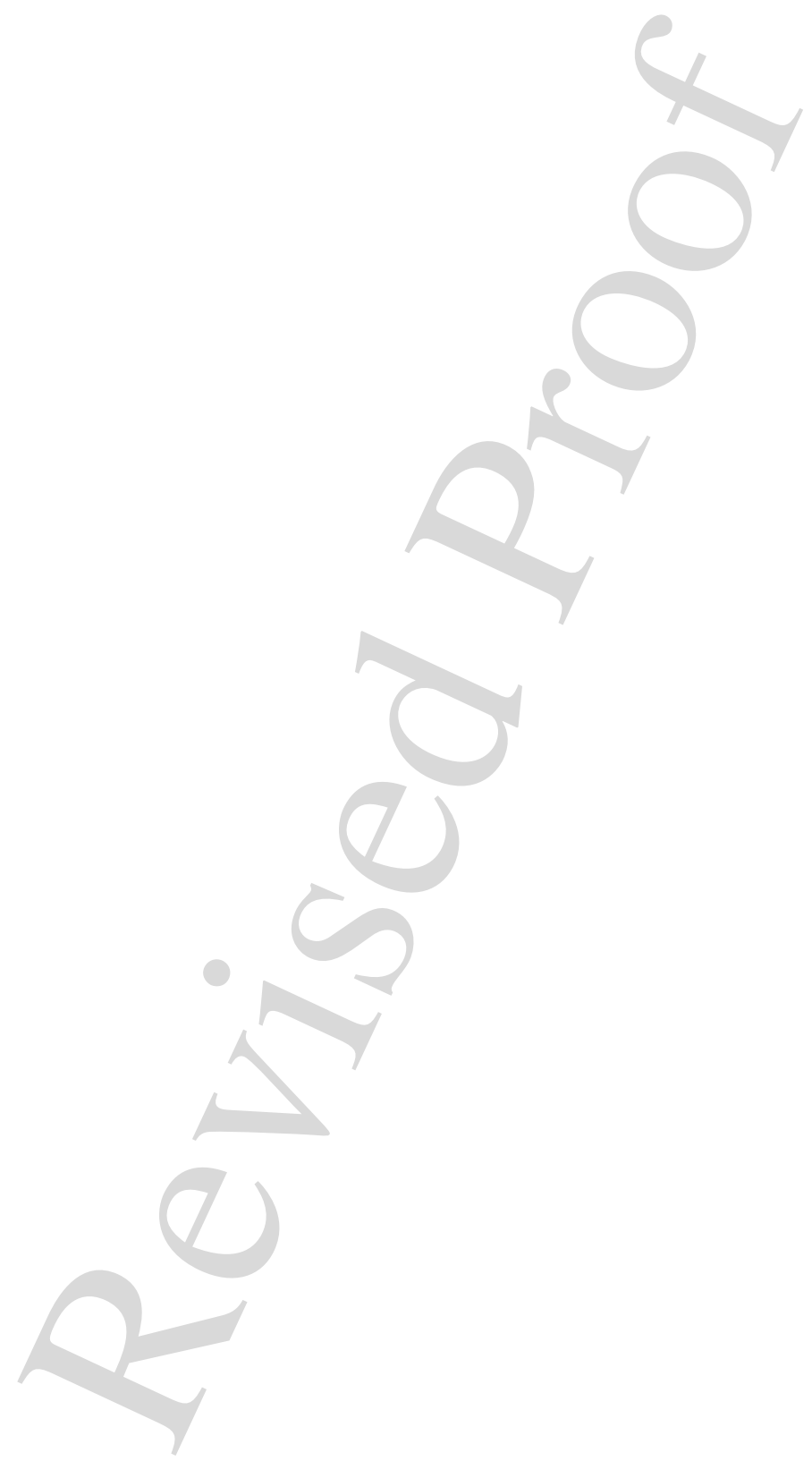

照 Springer

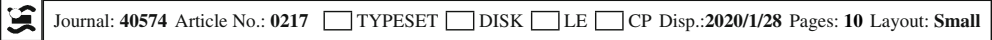

